\title{
两步晶化制备高性能 $\boldsymbol{a} \& \boldsymbol{b}$ 取向 $\mathbf{T}$ 型沸石膜
}

\author{
张 博 $^{1}$, 张 宁 ${ }^{1}$, 杨建华 ${ }^{2}$, 兰建成 ${ }^{2}$, 王金渠 ${ }^{1,2}$
}

(1. 大连理工大学 石油与化学工程学院, 盘锦 $124221 ; 2$. 大连理工大学 精细化工国家重点实验室, 吸附与无机 膜研究所, 大连 116024)

摘 要: 采用廉价的大孔 $\alpha-\mathrm{Al}_{2} \mathrm{O}_{3}$ 作为载体, 通过二次晶种诱导+两步变温水热合成工艺, 成功制备出薄而致密的高 性能 $\mathrm{T}$ 型沸石膜。该方法能够充分发挥晶种的诱导成核作用，通过改变两阶段水热晶化温度和时间来控制晶种外 延生长和晶体生长方向, 最终获得了连续、无缺陷的 $a \& b$ 取向 $\mathrm{T}$ 型沸石膜。实验过程中详细考察了第一阶段的晶 化温度、晶化时间以及第二阶段的晶化温度对沸石膜表面结构和性能的影响，并将最优两步晶化条件下制备的膜用 于 $90 \mathrm{wt} \%$ 的异丙醇/水渗透汽化分离, 在 $75{ }^{\circ} \mathrm{C}$ 下膜的通量为 $3.84 \mathrm{~kg} \cdot \mathrm{m}^{-2} \cdot \mathrm{h}^{-1}$, 分离因子大于 10000 。

关 键 词: $\mathrm{T}$ 型沸石膜; 两步晶化; $a \& b$ 取向; 渗透汽化

中图分类号: TQ174 文献标识码: A

\section{High Performance $a \& b$ Oriented T Zeolite Membrane by a Two-stage Crystallization Synthesis}

\author{
ZHANG Bo $^{1}$, ZHANG Ning $^{1}$, YANG Jianhua ${ }^{2}$, LAN Jiancheng ${ }^{2}$, WANG Jinqu ${ }^{1,2}$
}

(1. School of Petroleum and Chemical Engineering, Dalian University of Technology, Panjin124221, China; 2. Institute of Adsorption and Inorganic Membrane, State Key Laboratory of Fine Chemicals, Dalian University of Technology, Dalian 116024, China)

\begin{abstract}
A thin and dense high-performance T-type zeolite membrane was successfully prepared by a two-step seed crystal induction plus two-step temperature-varied hydrothermal synthesis on inexpensive and macroporous $\alpha$ - $\mathrm{Al}_{2} \mathrm{O}_{3}$ support. This method can fully perform nucleation of seed crystal, regulate the epitaxial growth and crystal growth direction by changing the hydrothermal crystallization temperature and time during the two-stage. Finally a continuous and defect-free $a \& b$ oriented zeolite T membrane was obtained. Effects of crystallization temperature and crystallization time of the first-stage and crystallization temperature of the second-stage on the surface structure and properties of zeolite membranes were investigated. The T-type zeolite membrane prepared under the optimal two-step crystallization condition displayed high pervaporation performance with flux over $3.84 \mathrm{~kg} \cdot \mathrm{m}^{-2} \cdot \mathrm{h}^{-1}$ and separation factor higher than 10000 for separation of $90 \mathrm{wt} \%$ isopropanol/water at $75{ }^{\circ} \mathrm{C}$.
\end{abstract}

Key words: T zeolite membrane; two-stage crystallization; $a \& b$ orientation; pervaporation

具有四面体结构的沸石膜作为一类新型无机微 孔膜 ${ }^{[1]}$, 有着独特的孔道结构、化学稳定性好、耐高
温、机械强度高等优点 ${ }^{[2-3]}$ ，在有机物分离方面比聚 合物膜有更大的潜力 ${ }^{[4]}$ 。与常规蒸馏相比，使用沸石

收稿日期：2019-09-10；收到修改稿日期：2019-11-01

基金项目：国家高新技术研究发展计划(863 计划)(2015AA03A602)

National High Technology and Development Program of China (2015AA03A602)

作者简介: 张 博(1995-), 女, 硕士研究生. E-mail: zb21727009@mail.dlut.edu.cn ZHANG Bo(1995-), female, Master candidate. E-mail: zb21727009@mail.dlut.edu.cn

通讯作者: 王金渠，教授. E-mail: wjinqu@dlut.edu.cn WANG Jinqu, professor. E-mail: wjinqu@dlut.edu.cn 
膜的渗透蒸发 $(\mathrm{PV})$ 具有低能耗的优点, 在有机溶剂 的分离中引起了越来越多的关注, 特别是对于共沸 和近沸混合物的分离 ${ }^{[5-7]}$ 。

$\mathrm{T}$ 沸石是毛沸石和菱钾沸石交互生长的一类具 有四面体结构的共生沸石, $\mathrm{Si} / \mathrm{Al}$ 为 3 4, 同时具有 较好的亲水性和耐酸性。 $\mathrm{T}$ 沸石框架中毛沸石片的 堆垛层错, 渗透的有效孔径是毛沸石 $(0.36 \mathrm{~nm} \times$ $0.51 \mathrm{~nm})$ 的孔径, 其孔径介于水和大多数有机物之 间, 因此 $\mathrm{T}$ 型沸石膜在有机溶剂脱水方面具有广阔 的应用前景 ${ }^{[8-9]}$ 。

近年来, 人们越来越关注具有可控微观结构沸 石膜的制备，如控制晶体取向和表面粗糙度 ${ }^{[10-11]}$ 。 方面沸石晶体中纳米尺寸孔道的优先取向可以改善 分子的质量传递行为; 另一方面通过晶体的有序排 列可以减少晶界缺陷, 从而提高分离性能 ${ }^{[10,12-14]}$ 。 而且有研究表明, 高度取向的沸石膜比随机取向的 沸石膜具有更好的耐腐蚀性 ${ }^{[15]}$ 和机械性能 ${ }^{[16]}$ 。目前 已经成功合成了多种取向性沸石膜, 包括 $\mathrm{MFI}^{[10-11,16-17]}, \mathrm{MOF}^{[12-13]}, \mathrm{DDR}^{[18]}$ 等。多数情况下, $\mathrm{T}$ 沸石为长棒状晶体, 沿 $c$ 轴的晶体尺寸最长, 因此 侧面(平行于 $c$ 轴)的面积明显大于基面(垂直于 $c$ 轴) 的面积, 所以当 $\mathrm{T}$ 沸石晶体在载体表面以 $a \& b$ 取向 生长时, 其覆盖率更大, 共生性更好, 而且分子扩 散路径更短。 $\mathrm{T}$ 沸石晶体是两种晶体的共生晶体, 受 多种因素影响, 其生长方向较难控制, 所以关于取 向性 $\mathrm{T}$ 型沸石膜的报道较少。周汉等 ${ }^{[19-20]}$ 通过微波 辅助传统加热的方法制备 $a \& b$ 取向 $\mathrm{T}$ 型沸石膜, 用 于辅助酯化反应和异丙醇渗透汽化脱水, 在 $338 \mathrm{~K}$ 下分离 $90 \mathrm{wt} \%$ 的异丙醇/水混合物, 通量和分离系数 分别为 $2.15 \mathrm{~kg} \cdot \mathrm{m}^{-2} \cdot \mathrm{h}^{-1}$ 和 10000 。

在 $\mathrm{T}$ 型沸石膜的研究报道中, 一步晶化法制备 的随机取向的沸石膜在一定程度上限制了膜的通量, 并且导致形成晶间孔。本研究采用廉价的大孔 $\alpha-\mathrm{Al}_{2} \mathrm{O}_{3}$ 作为载体, 使用二次晶种诱导十两步变温晶 化的方法, 通过控制膜表面晶体生长制备 $a \& b$ 取向 $\mathrm{T}$ 型沸石膜, 用于渗透汽化分离 $90 \mathrm{wt} \%$ 异丙醇和水 混合物。

\section{1 实验方法}

\section{1 实验试剂和材料}

氢氧化钠(纯度 $\geqslant 96 \%$, 天津市科密欧化学试剂 有限公司); 氢氧化钾(纯度 $\geqslant 85 \%$, 国药集团化学试 剂有限公司); 偏铝酸钠 $\left(41 \mathrm{wt} \% \mathrm{Al}_{2} \mathrm{O}_{3}, 24.92 \mathrm{wt} \%\right.$ $\mathrm{Na}_{2} \mathrm{O}$, 国药集团化学试剂有限公司); 硅溶胶 (40wt\% Ludox AS-40, 60wt $\% \mathrm{H}_{2} \mathrm{O}$, Aldrich Co. Ltd);
四甲基氢氧化铵 $\left(25 \mathrm{wt} \% \mathrm{TMAOH}, 75 \mathrm{wt} \% \mathrm{H}_{2} \mathrm{O}\right.$, 阿 拉丁化学试剂); 去离子水(大连理工大学盘锦校区 石油与化学工程学院自制); $\alpha-\mathrm{Al}_{2} \mathrm{O}_{3}$ 管状载体(外径 $12 \mathrm{~mm}$, 内径 $8 \mathrm{~mm}$, 管长 $50 \mathrm{~mm}$, 平均孔径 3 4 $\mu \mathrm{m}$, 孔隙率 30\% 40\%, 广东佛山陶瓷研究所)。

\section{2 载体管预处理}

分别采用 800 目 $(19 \mu \mathrm{m})$ 和 1500 目 $(10 \mu \mathrm{m})$ 的砂 纸打磨载体管至表面光滑, 然后进行超声水洗。再 分别对载体管进行酸洗和碱洗, 并用去离子水洗至 中性。载体管充分干燥后, 放置在 $550{ }^{\circ} \mathrm{C}$ 的马弗炉 中煅烧 $6 \mathrm{~h}$, 其升温和降温速率均为 $1{ }^{\circ} \mathrm{C} / \mathrm{min}$ 。最后 将煅烧后的载体管进行测漏，烘干备用。

\section{3 晶种及晶种层的制备}

采用摩尔比为 $n\left(\mathrm{SiO}_{2}\right): n\left(\mathrm{Al}_{2} \mathrm{O}_{3}\right): n\left(\mathrm{Na}_{2} \mathrm{O}\right)$ : $n\left(\mathrm{~K}_{2} \mathrm{O}\right): n\left(\mathrm{H}_{2} \mathrm{O}\right): n(\mathrm{TMAOH})=18.2: 1: 4.2: 1.5: 212.7$ : 0.82 配置合成液, 在 $100{ }^{\circ} \mathrm{C}$ 下晶化 $72 \mathrm{~h}$ 制备 $\mathrm{T}$ 沸石 大晶种; 采用摩尔比为 $n\left(\mathrm{SiO}_{2}\right): n\left(\mathrm{Al}_{2} \mathrm{O}_{3}\right): n\left(\mathrm{Na}_{2} \mathrm{O}\right)$ : $n\left(\mathrm{~K}_{2} \mathrm{O}\right): n\left(\mathrm{H}_{2} \mathrm{O}\right): n(\mathrm{TMAOH})=18.2: 1: 4.2: 1.5: 212.7$ : 1.82 配置合成液, 在 $100{ }^{\circ} \mathrm{C}$ 下晶化 $48 \mathrm{~h}$ 制备 $\mathrm{T}$ 沸石 小晶种, 然后分别配置 $3 \mathrm{wt} \%$ 的大晶种液和 $0.8 \mathrm{wt} \%$ 的 小晶种液。制备大晶种层: 将载体管两端密封放入 $160{ }^{\circ} \mathrm{C}$ 的烘箱中预热 $3 \mathrm{~h}$, 然后垂直放入大晶种液中 浸渍 $25 \mathrm{~s}$ 后缓慢提拉出来，充分烘干后用脱脂棉将 载体管表面多余的大晶种擦去。制备小晶种层: 将大 晶种修饰后的载体管两端密封放入 $100{ }^{\circ} \mathrm{C}$ 的烘箱中 预热 $3 \mathrm{~h}$, 浸入小晶种液 $30 \mathrm{~s}$ 后垂直缓慢提拉出来, 室温过夜后在 60 、100 和 $180{ }^{\circ} \mathrm{C}$ 的烘箱中进行升温 固化。

\section{$1.4 \mathrm{~T}$ 型沸石膜的制备}

采用摩尔比为 $n\left(\mathrm{SiO}_{2}\right): n\left(\mathrm{Al}_{2} \mathrm{O}_{3}\right): n\left(\mathrm{Na}_{2} \mathrm{O}\right): n\left(\mathrm{~K}_{2} \mathrm{O}\right)$ : $n\left(\mathrm{H}_{2} \mathrm{O}\right)=20: 1: 5.2: 1.8: 600$ 配置膜的合成液。首先 将 $\mathrm{NaOH} 、 \mathrm{KOH}$ 和 $\mathrm{NaAlO}_{2}$ 混合搅拌至澄清, 然后以 $3 \mathrm{~s}$ 一滴的速度把硅溶胶加入到上述溶液中, 陈化 $24 \mathrm{~h}$ 得到合成液。将上述合成液倒入不锈钢反应釜, 放入带有涂晶后的载体管, 在一定温度下水热晶化。 晶化完成水洗烘干后再进行一步小晶种涂覆, 然后进 行第二步水热晶化, 将第二步晶化完成的膜用去离子 水洗至中性后充分干燥, 以备后续表征和性能测试。

\section{5 表征和测试}

采用 D/MAX-2400 X 射线衍射仪对大、小晶种 和膜进行晶型和结晶度表征。采用美国 FEI 公司 Nova Nano SEM 450 场发射扫描电子显微镜观察晶 种以及载体管、晶种管、沸石膜的表面和截面形貌。 采用自制的渗透汽化装置对膜进行 $90 \mathrm{wt} \%$ 异丙醇/水 溶液脱水测试, 透过侧产物采用上海天美 GC7900 色 
谱进行含量分析。

\section{2 结果与讨论}

\section{1 晶种层制备}

实验采用的 $\alpha-\mathrm{Al}_{2} \mathrm{O}_{3}$ 载体表面粗糙不平且存在 较大缺陷, 如图 1(a, b), 为了有利于 $\mathrm{T}$ 沸石晶体的 生长且充分发挥晶种的诱导作用，采用两步变温 热浸渍大小晶种涂覆 ${ }^{[21-23]}$ 的方法提高晶种密度。将 合成的 2 和 $0.4 \mu \mathrm{m}$ 的 $\mathrm{T}$ 型沸石分别作为大晶种和 小晶种，图 1(c,d)为大晶种和小晶种的 SEM 照片。 从 $\mathrm{T}$ 沸石大、小晶种的 XRD 图谱(图 2)可以发现, 大、小晶种为纯的 $\mathrm{T}$ 沸石晶相。图 1(e,f)为大晶种 修饰后载体的表面和截面 SEM 照片, 可以看出 $2 \mu \mathrm{m}$ 的大晶种能较好地填补载体大孔缺陷, 使载体 表面更平滑, 有利于小晶种的涂覆。图 $1(\mathrm{~g}, \mathrm{~h})$ 为低温 涂覆制备的小晶种层的表面和截面 SEM 照片, 小晶 种层非常薄, 晶种排列紧凑、连续, 无明显缺陷。
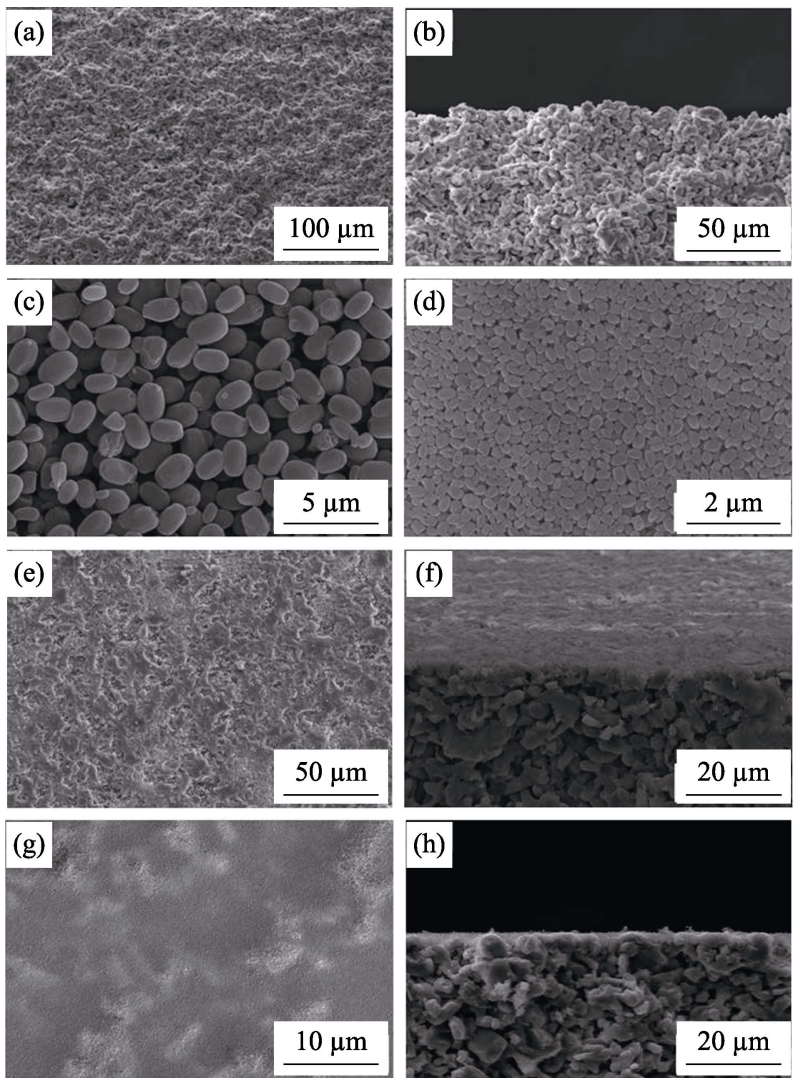

图 $1 \alpha-\mathrm{Al}_{2} \mathrm{O}_{3}$ 载体((a)-表面, (b)-截面)、大晶种(c)、小晶种 (d)、大晶种修饰后的载体((e)-表面, (f)-截面)以及小晶种层 ((g)-表面, (h)-截面)的 SEM 照片

Fig. 1 SEM images of $\alpha-\mathrm{Al}_{2} \mathrm{O}_{3}$ support photographed from surface (a) and cross section (b), large seeds (c), small seeds (d), modified by large seeds photographed from surface (e) and cross section (f), and the small seed layer photographed from surface $(\mathrm{g})$ and cross section $(\mathrm{h})$

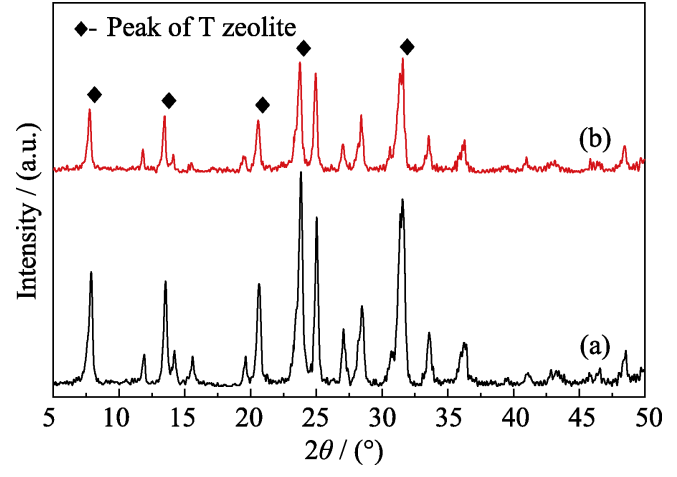

图 2 大晶种(a)和小晶种(b)的 XRD 图谱

Fig. 2 XRD patterns of large seeds (a) and small seeds (b)

\section{2 第一步晶化温度对沸石膜表面结构和性 能的影响}

分别在 110、120、130、135 和 $150{ }^{\circ} \mathrm{C}$ 下进行 第一步晶化制备 $\mathrm{T}$ 型沸石膜，其样品用 M1-1 M5-1 表示。图 3 为 $\mathrm{T}$ 型沸石膜 M1-1 M5-1 的表面和截 面 SEM 照片。由图 3(a,b) 可以看出, 膜 M1-1 基本 无 $\mathrm{T}$ 沸石晶体生成，小晶种层表面生成很多晶核， 此时还没有生成 $\mathrm{T}$ 型沸石膜。随着第一步晶化温度 的升高, 膜 M2-1(图 3(c, d)) 和 M3-1(图 3(e, f))逐渐 衍生出 $\mathrm{T}$ 沸石形貌，一部分小晶种开始外延生长成 为晶体, 晶核数量减少。这主要是由于在合成体系中, 晶核生成与晶体生长存在共生与竞争，而且低温有 利于晶核生成，同时高温有利于晶体生长 ${ }^{[24-25]}$ 。膜 M4-1(图 3(g, h)) 较为平整, 棒状 $\mathrm{T}$ 沸石晶体交互 “平躺”在载体表面, $a \& b$ 取向生长较好, 而且晶体 之间交互生长紧凑。从图 3(i,j)可以看出, 温度过 高使得膜层 M5-1 表面晶体开始倾向 $c$ 取向生长, 膜的平整度和致密性减弱。这种现象的原因是温度 对膜的取向生长有非常明显的作用, 在一定温度 范围内, 低温有利于膜 $a \& b$ 取向生长, 高温有利于 $c$ 取向生长 ${ }^{[26-27]}$ 。

第一步晶化后的沸石膜进行小晶种涂覆，一方 面填补第一步晶化过程形成的晶间缺陷和载体不平 引起的“凹陷区域”，另一方面发挥小晶种的诱导成 核作用 ${ }^{[22]}$ 和外延生长作用 ${ }^{[23]}$ 。图 4 为在不同的第一 步晶化温度下，两步晶化后形成的 $\mathrm{T}$ 型沸石膜 M1 M5 的表面和截面 SEM 照片。当第一步晶化温 度过低, 膜 M1(图 4(a,b))和 M2(图 4(c,d)) 表面晶体 基本由两次涂覆的小晶种外延生长而成, 短时间内 晶种外延生长程度较低, 晶体共生性较差。继续升 高温度, 晶体之间交互生长程度增大。膜 M4(图 4(g, h) ) 基本由 $a \& b$ 取向的棒状 $\mathrm{T}$ 沸石晶体交互生长而成, 膜厚约为 2 3 $\mu \mathrm{m}$ 。然而从图 4(i,j)中可以看出, 膜 层出现一些小的晶间孔, 这和图 3(i, j)高温下晶体 
倾向 $c$ 取向生长使膜层致密性减弱相一致。对比图 3 和图 4 可以发现, 第一步晶化的晶体 $a \& b$ 取向生长 程度越高, 沸石膜内层会越致密、平整, 有利于小晶 种在第二步过程中涂覆地更加均匀、连续, 从而提 供更多的成核位点。在第二步低温晶化过程中, 通 过充分发挥小晶种的外延生长作用和诱导成核作用, 进一步提高沸石膜的致密性和共生程度。

第二步涂覆的小晶种经过高温固化, 牢固地结 合在载体上，在第二步晶化过程中不易脱落、溶解。 合成液作为营养物质渗透过晶种层, $110{ }^{\circ} \mathrm{C}$ 下该小 晶种和第一步晶化后的晶体和晶核有助于形成新的 微晶, 这些新生微晶在强碱性合成介质中具有较高 的表面自由能, 可以有效提高沸石的晶化速率, 在 第二步晶化过程中, 晶种的外延生长和第一步晶化
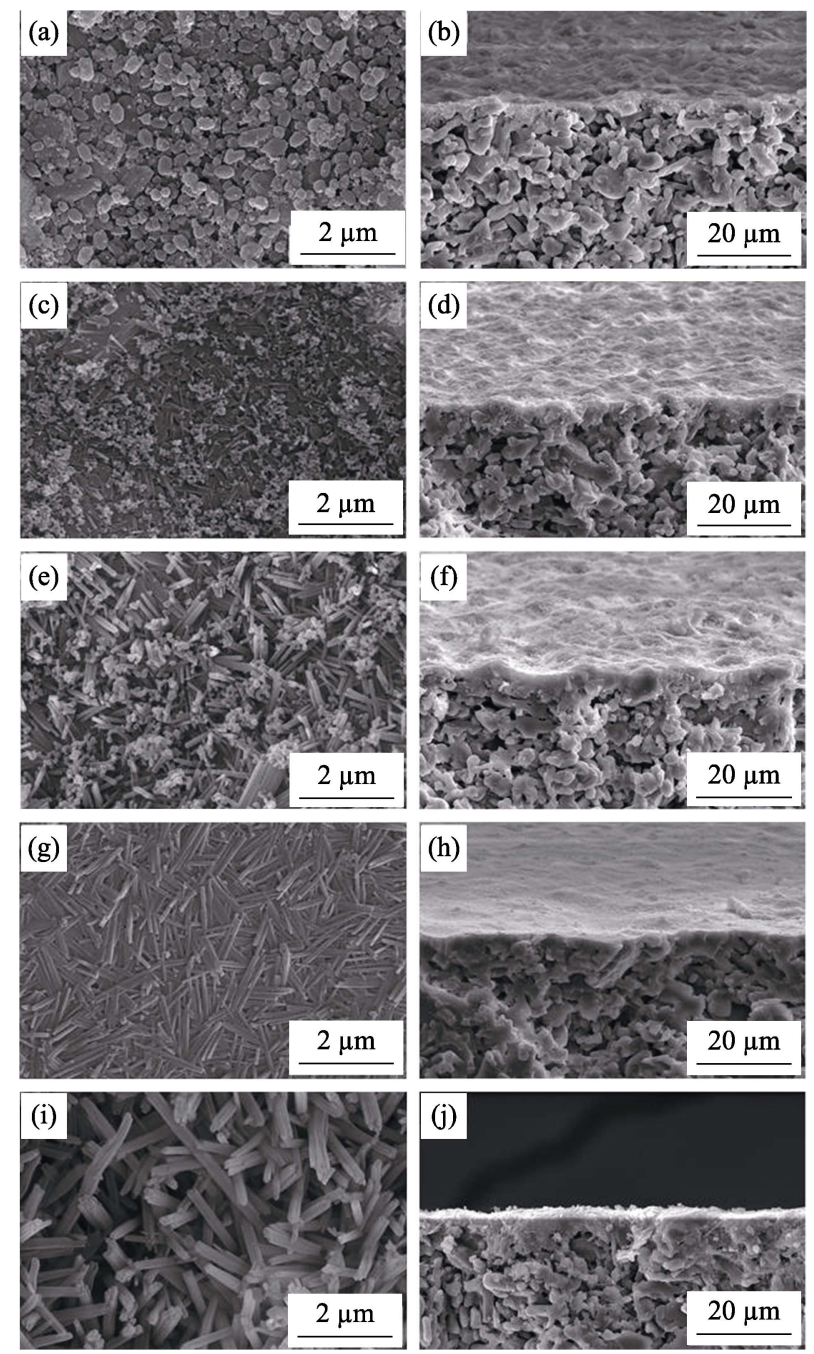

图 3 不同的第一步晶化温度下第一步晶化后的 $\mathrm{T}$ 型沸石膜 的 SEM 照片

Fig. 3 SEM images of T-type zeolite membranes after first-step crystallization prepared at different first-step crystallization temperatures

(a, b) M1-1, $110{ }^{\circ} \mathrm{C}$; (c, d) M2-1, $120{ }^{\circ} \mathrm{C}$; (e, f) M3-1, $130{ }^{\circ} \mathrm{C}$; (g, h) M4-1, $135{ }^{\circ} \mathrm{C}$; (i, j) M5-1, $150{ }^{\circ} \mathrm{C}$
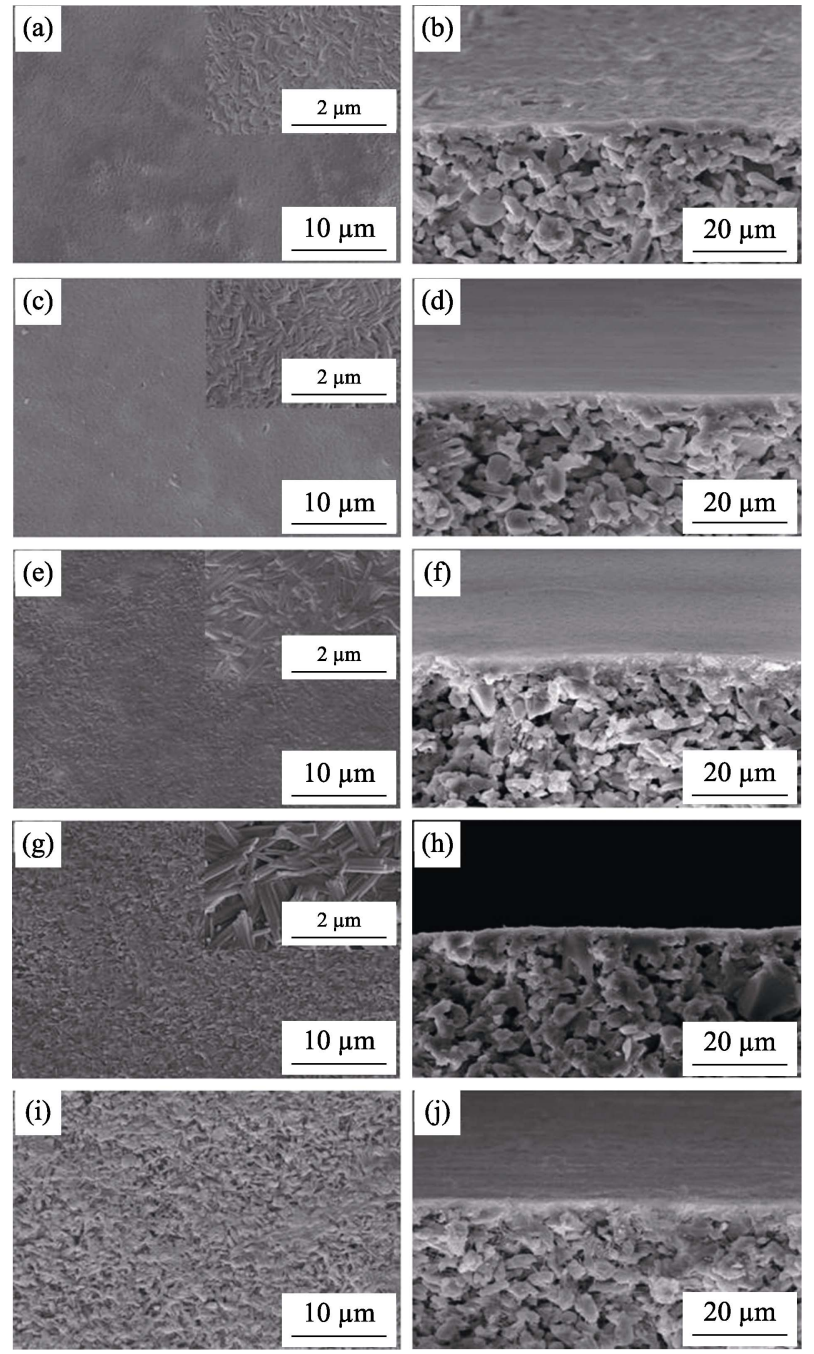

图 4 不同的第一步晶化温度下两步晶化后的 $\mathrm{T}$ 型沸石膜的 SEM 照片

Fig. 4 SEM images of T-type zeolite membranes after two-step crystallizations prepared at different first-step crystallization temperature

(a, b) M1, $110{ }^{\circ} \mathrm{C}$; (c, d) M2, $120{ }^{\circ} \mathrm{C}$; (e, f) M3, $130{ }^{\circ} \mathrm{C}$; (g, h) M4, $135{ }^{\circ} \mathrm{C}$; (i, j) M5, $150{ }^{\circ} \mathrm{C}$

后的晶体生长产生协同作用，短时间内制备出致密 的 $a \& b$ 取向 $\mathrm{T}$ 型沸石膜 ${ }^{[23-25,28]}$ 。

图 5 为 $\mathrm{T}$ 沸石膜 $\mathrm{M} 4$ 和 $\mathrm{T}$ 沸石小晶种的 XRD 图谱。从图 5(a)可以看出, $\mathrm{T}$ 沸石膜 $\mathrm{M} 4$ 的衍射峰由 载体峰和 $\mathrm{T}$ 沸石峰组成, 而且载体峰较强, 说明沸 石膜非常薄。根据 $\mathrm{T}$ 沸石的标准 XRD 图谱, 峰 (001)、(201)、(102)和(002)表示沿 $c$ 轴取向生长, 峰 (100)、(110)、(300)和(220)表示沿 $a$ 轴和 $b$ 轴取向 生长。将图 5(a, b)进行对比, (b)(膜 M4)中(001)、(201) 和(102)峰强度更弱, (100)、(110)和(220)峰强度更强, 而且 $I(300) / I(002)$ 由 0.32 (小晶种) 上升到 1.18 (膜 M4)。这些结果表明膜 M4 表面大部分 T 沸石晶体都 是 $a \& b$ 定向的，和周汉等 ${ }^{[19-20]}$ 的报道类似。

表 1 为 $\mathrm{T}$ 型沸石膜 M1 M5 在 $75{ }^{\circ} \mathrm{C}$ 下渗透蒸发 


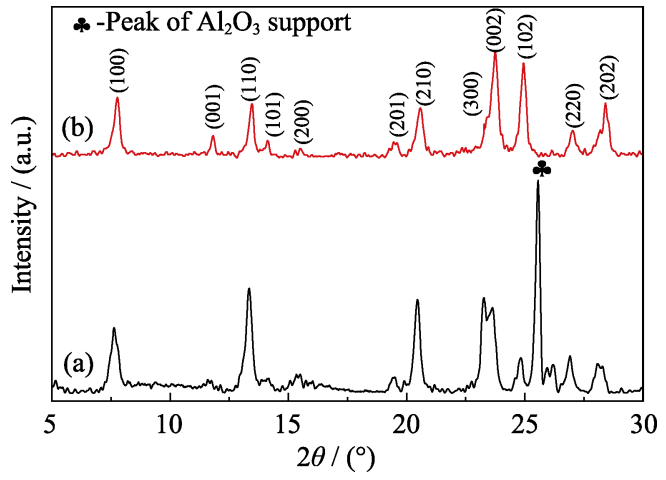

图 $5 \mathrm{~T}$ 沸石膜 M4(a)和 $\mathrm{T}$ 沸石小晶种(b)的 XRD 图谱

Fig. 5 XRD patterns of zeolite T membrane M4 (a) and small zeolite $\mathrm{T}$ seeds (b)

表 1 不同的第一步晶化温度制备的 $\mathbf{T}$ 型 沸石膜的渗透汽化性能

Table 1 Pervaporation performance of $T$ zeolite membranes prepared under different first-step crystallization temperatures

\begin{tabular}{|c|c|c|c|c|c|c|}
\hline \multirow[t]{2}{*}{ No. } & \multicolumn{2}{|c|}{$\begin{array}{c}\text { First-step } \\
\text { crystallization }\end{array}$} & \multicolumn{2}{|c|}{$\begin{array}{l}\text { Second-step } \\
\text { crystallization }\end{array}$} & \multirow{2}{*}{$\begin{array}{c}J / \\
\left(\mathrm{kg} \cdot \mathrm{m}^{-2} \cdot \mathrm{h}^{-1}\right)\end{array}$} & \multirow[t]{2}{*}{$\alpha$} \\
\hline & Temp. $/ /^{\circ} \mathrm{C}$ & Time/h & Temp. $/{ }^{\circ} \mathrm{C}$ & Time/h & & \\
\hline M1 & 110 & 4 & 110 & 6 & - & - \\
\hline M2 & 120 & 4 & 110 & 6 & 4.19 & 968 \\
\hline M3 & 130 & 4 & 110 & 6 & 3.87 & 5219 \\
\hline M4 & 135 & 4 & 110 & 6 & 3.84 & $>10000$ \\
\hline M5 & 150 & 4 & 110 & 6 & 3.53 & 2918 \\
\hline
\end{tabular}

$J$ : Pervaporation flux; $\alpha$ : Separation factor

Pervaporation conditions: $90 \mathrm{wt} \%$ isopropanol/ $/ \mathrm{H}_{2} \mathrm{O}, 75{ }^{\circ} \mathrm{C}$

分离 $90 \mathrm{wt} \%$ 异丙醇/水的性能结果, 表明第一步晶化温 度过高或者过低都不利于高性能 $\mathrm{T}$ 型沸石膜的形成, $135^{\circ} \mathrm{C}$ 下制备的膜 $\mathrm{M} 4$ 性能最好, 通量为 $3.84 \mathrm{~kg} \cdot \mathrm{m}^{-2} \cdot \mathrm{h}^{-1}$, 分离系数大于 10000 。

\section{3 第一步晶化时间对沸石膜表面结构和性 能的影响}

在第一阶段的合成过程中, 继续探究第一步晶 化时间对沸石膜表面结构和性能的影响。图 6 为在 不同的第一步晶化时间下, 第一步晶化后的 $\mathrm{T}$ 型沸石 膜 M6-1 M9-1 的表面和截面 SEM 照片。由图 6(a, b) 可以看出, 膜 M6-1 表面小晶种开始有外延生长的 趋势, 但是还没有 $\mathrm{T}$ 沸石晶体生成。当第一步晶化 时间延长至 $3 \mathrm{~h}$, 膜 M7-1(图 6(c,d)) 开始出现棒状 T 沸石晶相, 但是晶体生长程度较低。随着第一步晶 化时间的延长，膜 M8-1(图 6(e,f))、M9-1(图 6(g,h)) 表面由随机取向分布的晶体组成, 膜层疏松且不平 整, 这主要是由于 $\mathrm{T}$ 沸石晶体在晶化时间较长时不 定向生长较明显 ${ }^{[24]}$ 。

图 7 为改变第一步晶化时间, 两步晶化后形成的
$\mathrm{T}$ 型沸石膜 M6 M9 的表面和截面 SEM 照片。当第一 步晶化时间较短时, 膜 M6(图 7(a, b)) 和 M7(图 7(c, d)) 表面晶体主要由两次涂覆的小晶种在第二阶段外延 生长而成, 有部分载体裸露。延长第一步晶化时间 至 5 和 $6 \mathrm{~h}$, 膜 M8(图 7(e, f)) 和 M9(图 7(g, h)) 表面晶 体共生程度减弱。对比图 4(g, h), 膜 M4 表面晶体 尺寸最均匀, 生长方向基本一致的晶体使得膜层致 密性最好。从表 2 也可以看出, 随着第一步晶化时 间的延长, 沸石膜的分离系数先增大后减小, 沸石 膜 M4 展现了较好的渗透通量和分离系数。

\section{4 第二步晶化温度对沸石膜表面结构和性 能的影响}

图 8 为在不同的第二步晶化温度下, 两步晶化 后制备的 $\mathrm{T}$ 型沸石膜 M10 M13 的表面和截面 SEM 照片。从膜 M10(图 8(a,b))中可以看到小晶种之间 的界面, 此时由于温度过低还未有晶体生成。当第 二步晶化温度为 $100{ }^{\circ} \mathrm{C}$ 时, 膜 M11(图 8(c, d)) 表面 晶体生长程度明显增强, 但是仍然存在一些小的晶 间缺陷。随着第二步晶化温度的升高, 膜 M12 (图 8(e, f))和 M13(图 8(g,h)) 内部存在晶间孔，这主
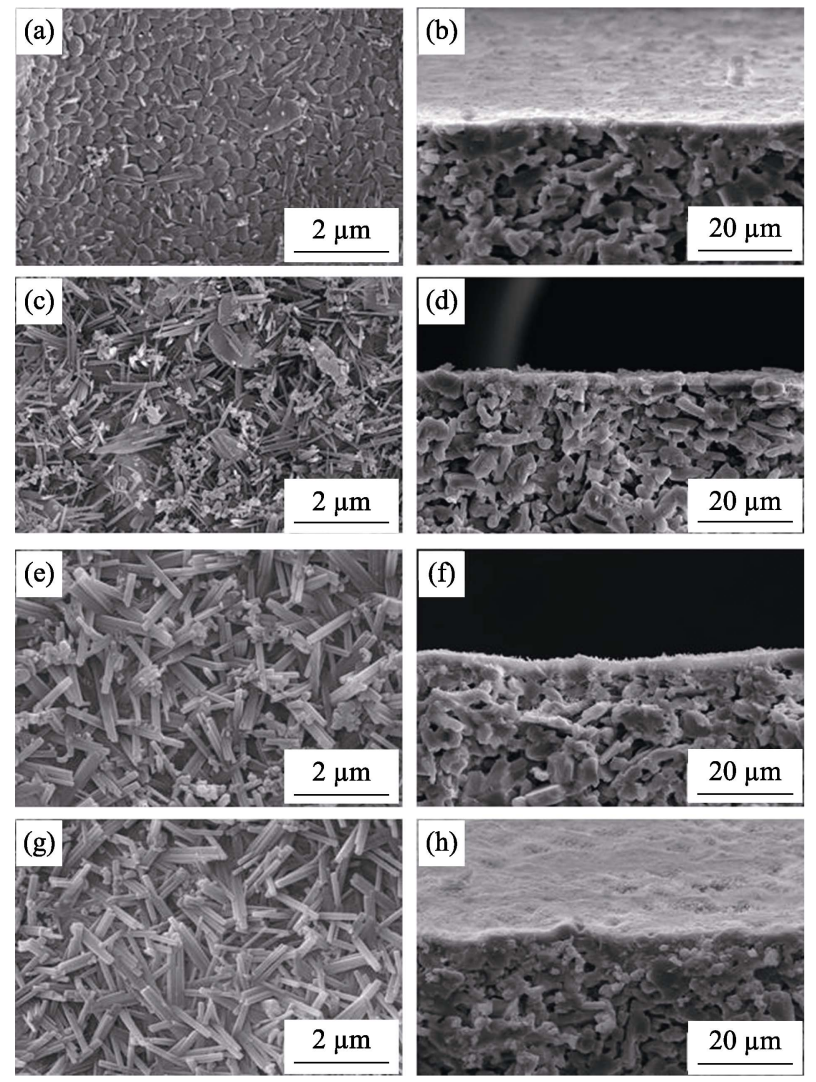

图 6 不同的第一步晶化时间下第一步晶化后的 $\mathrm{T}$ 型沸石膜 的 SEM 照片

Fig. 6 SEM images of T-type zeolite membranes after first-step crystallization prepared with varying first-step crystallization time

(a, b) M6-1, 2 h; (c, d) M7-1, 3 h; (e, f) M8-1, 5 h; (g, h) M9-1, 6 h 

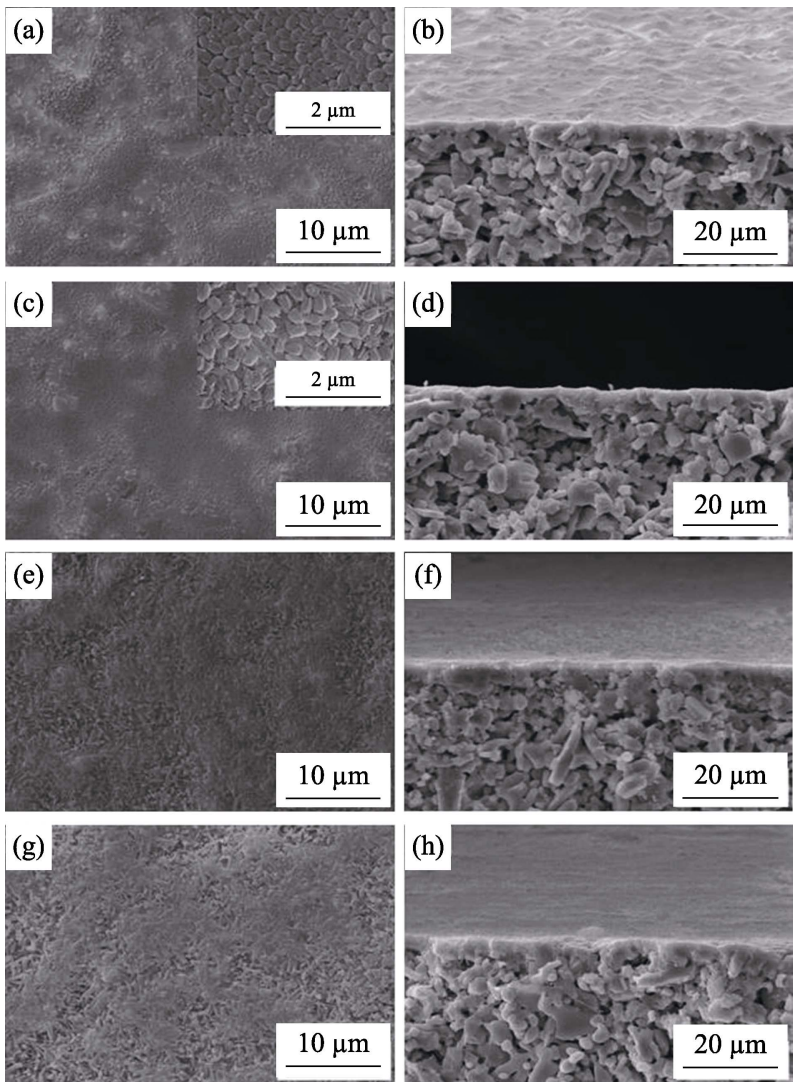

图 7 不同的第一步晶化时间下两步晶化后的 $\mathrm{T}$ 型沸石膜的 SEM 照片

Fig. 7 SEM images of T-type zeolite membranes after two-step crystallizations prepared with different first-step crystallization time

(a, b) M6, 2 h; (c, d) M7, 3 h; (e, f) M8, 5 h; (g, h) M9, 6 h

表 2 不同的第一步晶化时间制备的 $\mathbf{T}$ 型 沸石膜的渗透汽化性能

Table 2 Pervaporation performance of $T$ zeolite membranes prepared with different first-step crystallization time

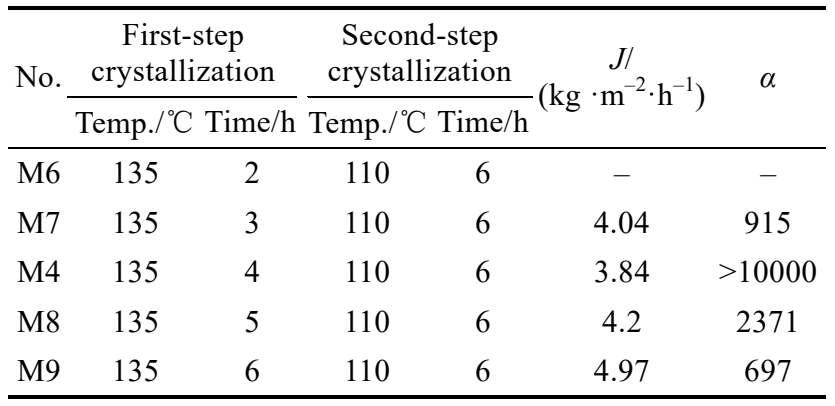

Pervaporation conditions: $90 \mathrm{wt} \%$ isopropanol $/ \mathrm{H}_{2} \mathrm{O}, 75{ }^{\circ} \mathrm{C}$

要是由于在较高温度下，第一阶段形成的 $\mathrm{T}$ 沸石晶 体开始倾向 $c$ 取向生长, 减弱了膜底层晶体的共生 性，从而降低了膜层的致密性。对比图 8 和图 $4(\mathrm{~g}, \mathrm{~h})$ 第二步晶化温度为 $110{ }^{\circ} \mathrm{C}$ 情况下膜 $\mathrm{M} 4$ 最致密，对 应表 3 也可以得出膜 M4 的渗透蒸发性能最佳，和 第一步晶化温度类似，第二步晶化温度过高或者过 低都会使膜的分离性能下降。
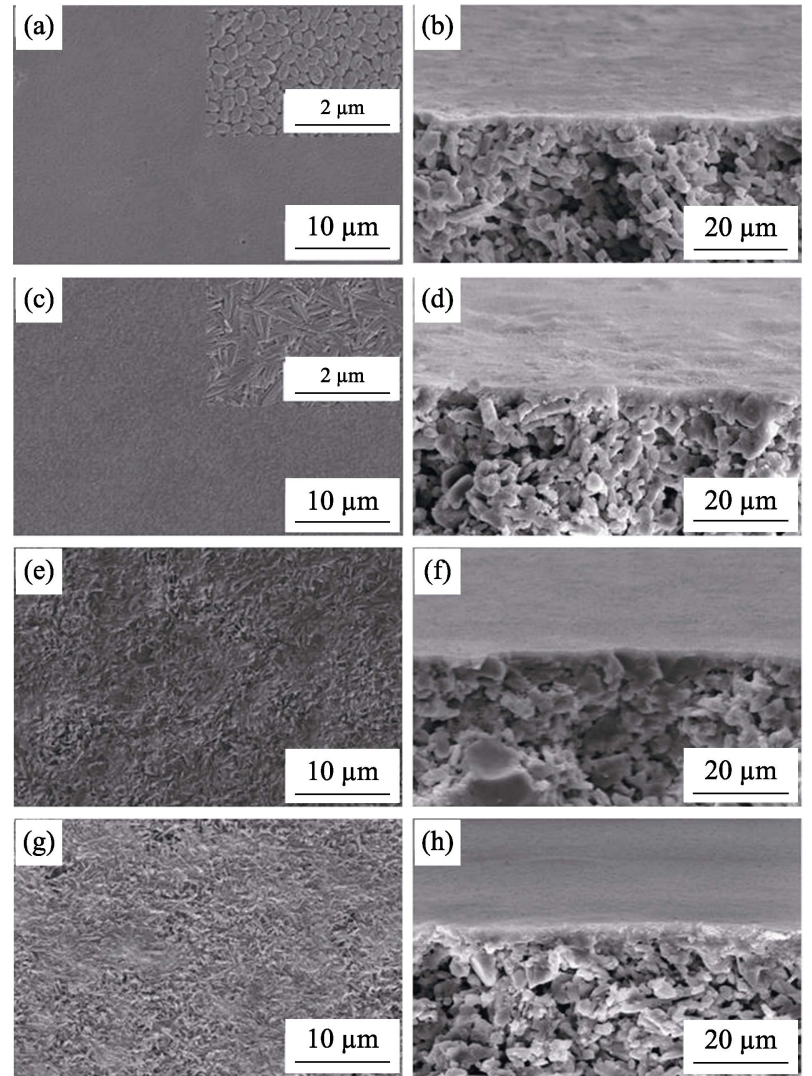

图 8 不同第二步晶化温度下两步晶化后的 $\mathrm{T}$ 型沸石膜的 SEM 照片

Fig. 8 SEM images of T-type zeolite membranes after two-step crystallizations prepared at different second-step crystallization temperatures

(a, b) M10, $90{ }^{\circ} \mathrm{C}$; (c, d) M11, $100{ }^{\circ} \mathrm{C}$; (e, f) M12, $120{ }^{\circ} \mathrm{C}$; (g, h) M13, $130{ }^{\circ} \mathrm{C}$

表 3 不同的第二步晶化温度制备的 $\mathbf{T}$ 型 沸石膜的渗透汽化性能

Table 3 Pervaporation performance of $\mathbf{T}$ zeolite membranes prepared under different second-step crystallization temperatures

\begin{tabular}{|c|c|c|c|c|c|c|}
\hline \multirow[t]{2}{*}{ No } & \multicolumn{2}{|c|}{$\begin{array}{c}\text { First-step } \\
\text { crystallization }\end{array}$} & \multicolumn{2}{|c|}{$\begin{array}{l}\text { Second-step } \\
\text { crystallization }\end{array}$} & $\begin{array}{c}J / \\
-2\end{array}$ & \multirow[t]{2}{*}{$\alpha$} \\
\hline & \multicolumn{2}{|c|}{$/{ }^{\circ} \mathrm{C}$ Tir } & emp. & Time/1 & & \\
\hline M10 & 135 & 4 & 90 & 6 & - & - \\
\hline M11 & 135 & 4 & 100 & 6 & 3.94 & 2646 \\
\hline M4 & 135 & 4 & 110 & 6 & 3.84 & $>10000$ \\
\hline M12 & 135 & 4 & 120 & 6 & 3.97 & 5867 \\
\hline M13 & 135 & 4 & 130 & 6 & 3.41 & 5594 \\
\hline
\end{tabular}

Pervaporation conditions: $90 \mathrm{wt} \%$ isopropanol $/ \mathrm{H}_{2} \mathrm{O}, 75{ }^{\circ} \mathrm{C}$

表 4 为 $\mathrm{T}$ 型沸石膜在 $75{ }^{\circ} \mathrm{C}$ 下用于 $90 \mathrm{wt} \%$ 异丙 醇/水体系分离的渗透蒸发性能。文献 $[21,23,29]$ 采用 一步水热晶化的方法制备了随机取向的 $\mathrm{T}$ 型沸石膜, 合成时间较长且渗透汽化通量较小。文献[19]采用 传统加热+微波加热的方法制备了 $7 \mu \mathrm{m}$ 厚的 $a \& b$ 定 向 $\mathrm{T}$ 型沸石膜, 渗透通量为 $2.15 \mathrm{~kg} \cdot \mathrm{m}^{-2} \cdot \mathrm{h}^{-1}$ 。本工作 
表 $4 \mathrm{~T}$ 型沸石膜用于 $75{ }^{\circ} \mathrm{C} 、 90 w t \%$

异丙醇/水体系分离的渗透蒸发性能

Table 4 Pervaporation performance of zeolite $T$ membranes for $90 \mathrm{wt} \%$ isopropanol/water mixture at $75{ }^{\circ} \mathrm{C}$

\begin{tabular}{cccccc}
\hline Support & $\begin{array}{c}\text { Temp./ } \\
{ }^{\circ} \mathrm{C}\end{array}$ & $\begin{array}{c}\text { Time/ } \\
\mathrm{h}\end{array}$ & $\begin{array}{c}J / \\
\left(\mathrm{kg} \cdot \mathrm{m}^{-2} \cdot \mathrm{h}^{-1}\right)\end{array}$ & $\alpha$ & Ref. \\
\hline$\alpha-\mathrm{Al}_{2} \mathrm{O}_{3}$ disc & 120 & 24 & 2.24 & 10000 & {$[29]$} \\
$\alpha-\mathrm{Al}_{2} \mathrm{O}_{3}$ tube & 100 & 30 & 2.52 & 10000 & {$[21]$} \\
$\alpha-\mathrm{Al}_{2} \mathrm{O}_{3}$ tube & 120 & 16 & 2.96 & 6400 & {$[23]$} \\
$\alpha-\mathrm{Al}_{2} \mathrm{O}_{3}$ tube $100+140^{a}$ & $8+1^{a}$ & 2.15 & 10000 & {$[19]$} \\
$\alpha-\mathrm{Al}_{2} \mathrm{O}_{3}$ tube $150+120^{b}$ & $4+8^{b}$ & 2.04 & 2874 & {$[30]$} \\
$\alpha-\mathrm{Al}_{2} \mathrm{O}_{3}$ tube $135+110^{c}$ & $4+6^{c}$ & 3.84 & $>10000$ This work \\
\hline$\alpha 100+140.8+1:$ & & &
\end{tabular}

a 100+140, 8+1: hydrothermal synthesis at $100{ }^{\circ} \mathrm{C}$ for $8 \mathrm{~h}$ followed by microwave synthesis at $140{ }^{\circ} \mathrm{C}$ for $1 \mathrm{~h}$

b 150+120, 4+8: hydrothermal synthesis at $150{ }^{\circ} \mathrm{C}$ for $4 \mathrm{~h}$ followed by hydrothermal synthesis at $120{ }^{\circ} \mathrm{C}$ for $8 \mathrm{~h}$ c 135+110, 4+6: hydrothermal synthesis at $135{ }^{\circ} \mathrm{C}$ for $4 \mathrm{~h}$ followed by hydrothermal synthesis at $110{ }^{\circ} \mathrm{C}$ for $6 \mathrm{~h}$

采用两步涂晶+两步传统水热的方法，一方面，小 晶种两步涂覆可以减少晶间缺陷, 提高沸石膜的致 密程度; 另一方面, 减少了消耗的能量, 过快的微 波加热速率对晶体生长速率和生长方向较难控制, 传统烘箱加热可以避免这些影响, 制备的沸石膜厚 度降低至 2 3 $\mu \mathrm{m}$, 渗透通量达到 $3.84 \mathrm{~kg} \cdot \mathrm{m}^{-2} \cdot \mathrm{h}^{-1}$ 。与 文献相比, 本研究采用两步涂晶十两步水热晶化的 方法制备了 $a \& b$ 取向的 $\mathrm{T}$ 型沸石膜，其渗透通量高 于其它沸石膜, 且表现出较好的分离性能。

\section{3 结论}

采用两步晶化的方法, 在大孔 $\alpha-\mathrm{Al}_{2} \mathrm{O}_{3}$ 载体上 $135{ }^{\circ} \mathrm{C}$ 晶化 $4 \mathrm{~h}+110{ }^{\circ} \mathrm{C}$ 晶化 $6 \mathrm{~h}$ 成功制备出高性能 $a \& b$ 取向 $\mathrm{T}$ 型沸石膜。结果表明, 合理控制两阶段 的晶化时间和晶化温度是制备致密 $a \& b$ 取向 $\mathrm{T}$ 型沸 石膜的关键。改变两个阶段的晶化温度和时间能有 效控制晶种外延生长程度和方向以及新生晶核和晶 体生长方向, 从而制备出 2 3 $\mu \mathrm{m}$ 厚的 $a \& b$ 取向致 密 $\mathrm{T}$ 型沸石膜。将制备得到的 $\mathrm{T}$ 型沸石膜用于 $75{ }^{\circ} \mathrm{C}$ 下 $90 \mathrm{wt} \%$ 异丙醇/水分离, 渗透通量为 $3.84 \mathrm{~kg} \cdot \mathrm{m}^{-2} \cdot \mathrm{h}^{-1}$, 分离系数大于 10000 。

\section{参考文献:}

[1] JUSOH N, YEONG Y F, LAU K K, et al. Fabrication of silanated zeolite T/6FDA-durene composite membranes for $\mathrm{CO}_{2} / \mathrm{CH}_{4}$ separation. Journal of Cleaner Production, 2017, 166: 1043-1058.

[2] FARD A K, MCKAY G, BUEKENHOUDT A, et al. Inorganic membranes: preparation and application for water treatment and desalination. Materials, 2018, 11(1): 74 .
[3] BEDARD R, LIU C Q. Recent advances in zeolitic membranes. Annual Review of Materials Research, 2018, 48: 83-110.

[4] RANGNEKAR N, MITTAL N, ELYASSI B, et al. Zeolite membranesa review and comparison with MOFs. Chemical Society Reviews, 2015, 44(20): 7128-7154.

[5] JYOTHI M S, REDDY K R, SOONTARAPA K, et al. Membranes for dehydration of alcohols via pervaporation. Journal of Environmental Management, 2019, 242: 415-429.

[6] CAO Y, LI Y X, WANG M, et al. High-flux NaA zeolite pervaporation membranes dynamically synthesized on the alumina hollow fiber inner-surface in a continuous flow system. Journal of Membrane Science, 2019, 570: 445-454.

[7] ALOMAIR A A, AL-JUBOURI S M, HOLMES S M. A novel approach to fabricate zeolite membranes for pervaporation processes. Journal of Materials Chemistry A, 2015, 3(18): 9799-9806.

[8] LILLERUD K P, RAEDER J H. On the synthesis of erionite-offretite intergrowth zeolites. Zeolites, 1986, 6(6): 474-483.

[9] WANG X Q, XU R R. Studies on the transformation between erionite and offretite in T-type zeolite. Studies in Surface Science and Catalysis, 1985, 24: 111-118.

[10] AGRAWAL K V, TOPUZ B, PHAM T C T, et al. Oriented MFI membranes by gel-less secondary growth of sub-100 nm MFInanosheet seed layers. Advanced Materials, 2015, 27(21): 3243-3249.

[11] ZHOU M, KORELSKIY D, YE P C, et al. A uniformly oriented MFI membrane for improved $\mathrm{CO}_{2}$ separation. Angewandte ChemieInternational Edition, 2014, 53(13): 3492-3495.

[12] LI Y J, LIU H O, WANG H T, et al. GO-guided direct growth of highly oriented metal-organic framework nanosheet membranes for $\mathrm{H}_{2} / \mathrm{CO}_{2}$ separation. Chemical Science, 2018, 9(17): 4132-4141.

[13] SUN Y W, LIU Y, CARO J, et al. In-plane epitaxial growth of highly c-oriented $\mathrm{NH}_{2}$-MIL-125(Ti) membranes with superior $\mathrm{H}_{2} / \mathrm{CO}_{2}$ selectivity. Angewandte Chemie-International Edition, 2018, 57(49): 16088-16093.

[14] HUANG P S, LAM C H, SU C Y, et al. Scalable wet deposition of zeolite AEI with a high degree of preferred crystal orientation. Angewandte Chemie-International Edition, 2018, 57(40): 13271-13276.

[15] CAI R, SUN M W, CHEN Z W, et al. Ionothermal synthesis of oriented zeolite AEL films and their application as corrosion-resistant coatings. Angewandte Chemie-International Edition, 2008, 47(3): $525-528$.

[16] LI Z J, JOHNSON M C, SUN M W, et al. Mechanical and dielectric properties of pure-silica-zeolite low- $k$ materials. Angewandte Chemie-International Edition, 2006, 45(38): 6329-6332.

[17] NIAN P, YU T, SU M H, et al. Fabrication of $b$-oriented MFI film via Langmuir-Blodgett technique. Journal of Inorganic Materials, 2016, 31(4): 377-382.

[18] KIM E, HONG S, JANG E, et al. An oriented, siliceous deca-dodecasil 3R(DDR) zeolite film for effective carbon capture: insight into its hydrophobic effect. Journal of Materials Chemistry A, 2017, 5(22): 11246-11254.

[19] ZHOU H, LI Y S, ZHU G Q, et al. Microwave-assisted hydrothermal synthesis of $a \& b$-oriented zeolite $\mathrm{T}$ membranes and their pervaporation properties. Separation and Purification Technology, 2009, 65(2): 164-172.

[20] ZHOU H, LI Y S, ZHU G Q, et al. Microwave synthesis of $a \& b$ oriented zeolite $\mathrm{T}$ membranes and their application in pervaporation-assisted esterification. Chinese Journal of Catalysis, 2008, 29(7): 592-594.

[21] CHEN X X, WANG J Q, YIN D H, et al. High-performance zeolite $\mathrm{T}$ membrane for dehydration of organics by a new varying temperature hot-dip coating method. Aiche Journal, 2013, 59(3): 936-947.

[22] HUANG P, ZHOU L, LI H Z, et al. Preparation and characteriza- 
tion of high performance MFI zeolite membrane in ultradilute solution. Journal of Inorganic Materials, 2018, 33(3): 345-351.

[23] LYU Y J, LI H Z, YANG J H, et al. Preparation and characterization of high performance zeolite $\mathrm{T}$ membranes from clear solutions. Journal of Inorganic Materials, 2016, 31(7): 705-710.

[24] ZHANG X L, QIU L F, DING M Z et al. Preparation of zeolite T membranes by a two-step temperature process for $\mathrm{CO}_{2}$ separation. Industrial \& Engineering Chemistry Research, 2013, 52(46): 16364-16374.

[25] MEHDIPOURGHAZI M, MOHEB A, KAZEMIAN H. Incorporation of boron into nano-size MFI zeolite structure using a novel microwave-assisted two-stage varying temperatures hydrothermal synthesis. Microporous and Mesoporous Materials, 2010, 136(1/2/3): $18-24$.

[26] ZHANG F, HU N, ZHOU R F, et al. Preparation of Zeolite T Membrane and Its Application in Esterification. Chinese Science and Technology Reporting Conference, Beijing, 2010: 537-540.

[27] ZHOU R F, HU L L, ZHANG Y J, et al. Synthesis of oriented zeolite $\mathrm{T}$ membranes from clear solutions and their pervaporation properties. Microporous and Mesoporous Materials, 2013, 174: $81-89$.

[28] LI G, KIKUCHI E, MASTUKATA M. ZSM-5 zeolite membranes prepared from a clear template-free solution. Microporous and Mesoporous Materials, 2003, 60(1/2/3): 225-235.

[29] SHAFIEI K, PAKDEHI S G, MOGHADDAM M K, et al. Improvement of zeolite $\mathrm{T}$ membrane via clear solution gel in dehydration of methanol, ethanol and 2-propanol. Separation Science and Technology, 2014, 49(6): 797-802.

[30] XU Z X, WU H D, ZHOU Z H, et al. Preparation of high performance zeolite $\mathrm{T}$ membranes by a two-stage crystallization method. Petrochemical Technology, 2017, 46(11): 1403-1408. 